\title{
Comparison between optical measurements made from natural and frozen samples at optical clearing
}

\author{
Luís Oliveira $^{* a, b}$, Maria Inês Carvalho ${ }^{c}$, Elisabete Nogueira ${ }^{a}$, Valery V. Tuchin ${ }^{\text {d, ef }}$ \\ ${ }^{a}$ Physics Department - Polytechnic of Porto, School of Engineering, Rua Dr. António Bernardino de \\ Almeida, 431, 4200-072 Porto, Portugal; \\ ${ }^{\mathrm{b}} \mathrm{PhD}$ student at FEUP - University of Porto, Rua Dr. Roberto Frias, 4200-465 Porto, Portugal; \\ ${ }^{c}$ DEEC/FEUP and INESC TEC, University of Porto, Rua Dr. Roberto Frias, 4200-465 Porto, \\ Portugal; \\ ${ }^{d}$ Research-Educational Institute of Optics and Biophotonics, Saratov State University, 83 \\ Astraskhanskaya str., Saratov 410012, Russia; \\ ${ }^{\mathrm{e}}$ Laboratory of Laser Diagnostics of Technical and Living Systems, Institute of Precise Mechanics \\ and Control RAS, Saratov 410028, Russia; \\ ${ }^{\mathrm{f}}$ Optoelectronics and Measurement Techniques Laboratory, P. O. Box 4500, University of Oulu, \\ FIN-90014, Oulu, Finland
}

\begin{abstract}
To determine the differences between the optical clearing effects created by ethylene glycol in fresh and frozen samples, we have performed several measurements from samples in both conditions. Fresh samples were used after animal sacrifice and frozen samples were kept at $-20^{\circ} \mathrm{C}$ for 72 hours. The different measurements performed with samples from both cases were total transmittance, collimated transmittance, total reflectance and specular reflectance. Considering, for instance, collimated transmittance measurements, we have verified that the spectra measured from both samples before adding the solution present different levels of collimated transmittance. The time-dependence evolution of the collimated transmittance spectrum is similar between both cases of samples, but since they present different levels of "natural" transmittance, the optical clearing effect is observed at different levels if we compare between fresh and frozen samples.
\end{abstract}

Keywords: Optical clearing, Optical transmittance, Optical reflectance, Absorbance.

\section{INTRODUCTION}

To develop and improve optical based clinical techniques it is very important to perform optical measurements from tissue samples to evaluate tissue response to optical stimulation.

Considering ex vivo tissue samples, we can measure optical transmittance, optical reflectance, absorbance, etc. ${ }^{1}$. Depending on the situation, such measurements can be made from fresh or frozen samples. Furthermore, if these measurements are performed while tissue samples undergo optical clearing treatments, the time-dependence of the measurements can be obtained to characterize the optical clearing treatment. Imaging techniques can also be used to study the optical clearing effect ${ }^{2,3}$. When immersing biological tissues in sugars, alcohols or their combinations, the optical clearing effect is created through the mechanisms of tissue dehydration and refractive index matching as a consequence of agent diffusion into the tissue ${ }^{4,5}$.

With the objective of studying the differences between optical clearing effects created in fresh and frozen ex vivo samples, we have performed some sets of measurements in skeletal muscle. The skeletal muscle can be described as a distribution of muscle fiber bundles in interstitial fluid. It has been verified that rat skeletal muscle contains approximately $75 \%$ of water ${ }^{6}$ with a low refractive index (1.333). The other $25 \%$ of the muscle represents the dried tissue, which is composed by filaments of actin and myosin, having a higher refractive index (1.584) ${ }^{7}$. Considering such constitution of the skeletal muscle, we see that a step refractive index profile exists, which causes considerable part of

Saratov Fall Meeting 2012: Optical Technologies in Biophysics and Medicine XIV; and Laser Physics and Photonics XIV,

Valery V. Tuchin, Elina A. Genina, Vladimir L. Derbov, Igor V. Meglinski, Eds., Proc. of SPIE Vol. 8699, 86990A

(C) 2013 SPIE · CCC code: 1605-742/13/\$18 - doi: 10.1117/12.2016936

Proc. of SPIE Vol. 8699 86990A-1 
light scattering in the muscle. The magnitude of that refractive index step can be significantly decreased with an optical clearing treatment as we have already verified in a previous study at collimated transmittance measurements ${ }^{7}$.

In the present study we have used fresh and frozen ex vivo muscle samples to study the variations of total transmittance $\left(T_{t}\right)$, collimated transmittance $\left(T_{c}\right)$, total reflectance $\left(R_{t}\right)$ and specular reflectance $\left(R_{s}\right)$ while under treatment with ethylene glycol. We have also used these measurements to calculate the variations of tissue absorbance $(A)$ and diffuse reflectance $\left(R_{d}\right)$ to compare between samples. From these measurements, we could observe the variations caused by optical clearing treatment and identify differences between the cases of fresh and frozen samples.

\section{EXPERIMENTAL METHOD}

The tissue samples, optical clearing agent, measuring assemblies and experimental methodology will be described here. In all the measuring setups described in subsections below, we have used a Tungsten Halogen lamp with a broad spectrum and a spectrometer to measure the spectra. The lamp is the HL-2000 model and the spectrometer is the AvaSpec-2048-USB2 model with UA grating set for 200-1100 nm and $50 \mu \mathrm{m}$ slit, both from Avantes Corporation.

The experimental methodology used with all samples (fresh and frozen) consisted on performing four sequential sets of measurements while the samples were submitted to optical clearing. The four sets of measurements were: $T_{t}, R_{t}, T_{c}$ and $R_{s}$. All the experimental setups used to perform these measurements will be presented and described in subsections below. We have started our first study with the fresh samples.

For each particular measurement assembly, we measured the light reference spectrum and the spectra from natural tissue and while tissue is under optical clearing treatment using the Scan mode of the spectrometer. Considering that the light reference is $100 \%$ of the light used in each experiment, we have calculated from the measurements the spectra of $T_{t}, R_{t}$, $T_{c}$ and $R_{s}$ by using equations 1,2,3 and 4 below. Additionally, we have obtained the $A$ and $R_{d}$ spectra for sequential time instants of the treatment by calculations with equations 5 and 6 below. The results obtained with all these equations are presented in percentage of the light reference used in each case. The following subsections contain an explanation for the individual calculations performed.

\subsection{Tissue samples}

The tissue samples used in our studies were obtained from the abdominal wall of the Wistar Han rat (see figure 1):

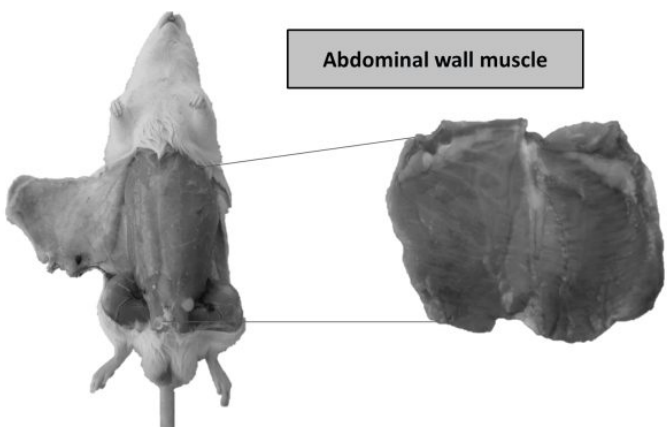

Figure 1: The abdominal wall muscle from Whistar Han rat.

From a single animal sacrifice, we have collected several samples with $0.5 \mathrm{~mm}$ thickness and circular section with approximately $1 \mathrm{~cm}$ of diameter ${ }^{5}$. From this collection of samples, we have considered two groups: fresh and frozen. Using the fresh samples, we immediately performed a set of measurements to obtain the response to light stimulation. The so called frozen samples were kept at $-20^{\circ} \mathrm{C}$ for 72 hours before being submitted to a similar study. In both cases the measurements were performed while samples were immersed in ethylene glycol which is a well known optical clearing agent $5,8,9,10$. The results obtained from the measurements with the two sets of samples were submitted to comparison so we could evaluate differences between the optical clearing effects created and this comparison is presented in section 3 . 
After comparing results from the two studies we can be sure that the fact of freezing the muscle samples for a period of 72 hours produces some changes in the natural responses of the muscle to light. Such changes can be identified both in transmittance and in reflectance measurements. Some particular differences between fresh and frozen samples were observed in the time dependence of some measurements. These differences will deserve our attention and explanation in sections 3 and 4 .

\subsection{Optical clearing agent - ethylene glycol}

The optical clearing agent that we have used in this study was ethylene glycol (EG). The solution of EG was prepared with $99 \%$ of this agent and $1 \%$ of water. We have measured the refractive index of this solution, obtaining the value of $1.4280^{5}$. EG is a powerful optical clearing agent as we could already observed in our previous studies ${ }^{5,8}$ and also as verified by other researchers $9,10,11,12$.

\subsection{Optical measurements}

In the present study, instead of measuring only the collimated transmittance as before ${ }^{\mathbf{5}}$, we have selected 4 types of measurements to perform a better characterization of the optical clearing effect. We will describe those measurements now. The measurement assemblies, corresponding measuring methodologies and calculations will be presented in the following sub-subsections.

\subsubsection{Total transmittance set-up}

To measure $T_{t}$ the sample is placed at the sample port of the integrating sphere (left entrance in figure 2) and light is introduced into the sphere through the sample. The optical signal proportional to totally transmitted light is collected at the exit port (lower hole in figure 2).

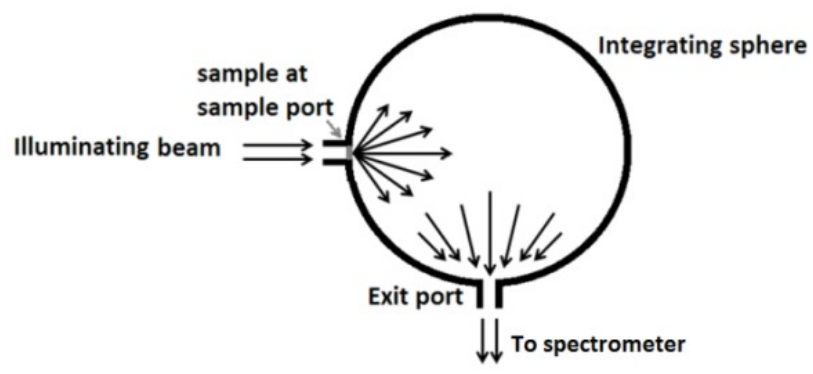

Figure 2: Total transmittance measuring assembly.

To obtain the $T_{t}$ spectra during the optical clearing of the tissue sample we measured first the spectrum of the light reference $\left(S_{t t}(\lambda)\right)$ and then the spectra of the total transmitted light through the sample at different time instants of the optical clearing process $\left(T_{t t}(\lambda, t)\right)$. Using these symbols we can use equation 1 to calculate the $T_{t}$ spectrum at any time $t$ :

$$
T_{t}(\lambda, t)=100 \% \times \frac{T_{t t}(\lambda, t)}{S_{t t}(\lambda)}
$$

\subsubsection{Total reflectance set-up}

To measure $R_{t}$ from the sample, illumination is now made through the illuminating port at $8^{\circ}$ with the normal direction to the sample (right side of the sphere in figure 3 ). The optical signal proportional to totally reflected light is collected at the exit port of the sphere (lower hole in figure 3 ). 
Figure 3: Total reflectance measuring assembly.

Using this assembly, we started by measuring the spectrum from the light reference $\left(S_{r t}(\lambda)\right)$. At different time instants of the optical clearing process, we measured the different spectra that are totally reflected from the muscle sample $\left(R_{r t}(\lambda, t)\right)$. Using these spectra, we calculate the $R_{t}$ spectra of the sample for any particular time of the treatment using equation 2 :

$$
R_{t}(\lambda, t)=100 \% \times \frac{R_{r t}(\lambda, t)}{S_{r t}(\lambda)}
$$

\subsubsection{Collimated transmittance set-up}

Figure 4 shows the simple assembly used to measure $T_{c}$ : A collimated beam $(\varnothing=6 \mathrm{~mm})$ is directed normally to the sample. Immediately before and after the sample two pinholes $(\varnothing=1 \mathrm{~mm})$ were placed to reduce the beam diameter. The transmitted beam was measured on the opposite side of the sample.

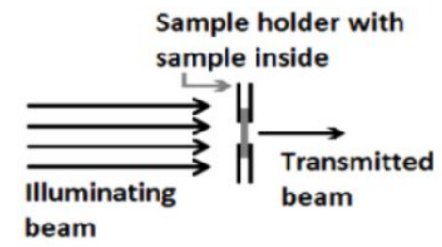

Figure 4: Collimated transmittance measuring assembly.

In this case, we consider $S_{t c}(\lambda)$ as the spectrum measured from the light reference and $T_{t c}(\lambda, t)$ as the collimated transmitted spectrum measured from the sample at a time $t$ of the treatment. Equation 3 allows the calculation of the $T_{c}$ spectrum of the sample at time $t$ :

$$
T_{c}(\lambda, t)=100 \% \times \frac{T_{t c}(\lambda, t)}{S_{t c}(\lambda)}
$$

\subsubsection{Specular reflectance set-up}

The measurement assembly to obtain $R_{s}$ (figure 5) is accordingly with the $R_{t}$ measurement assembly in angles and dimensions (figure 3 ). It uses an incident beam at $8^{\circ}$ with the normal direction to the sample surface and the reflected beam is also measured at the same angle on the other side. 


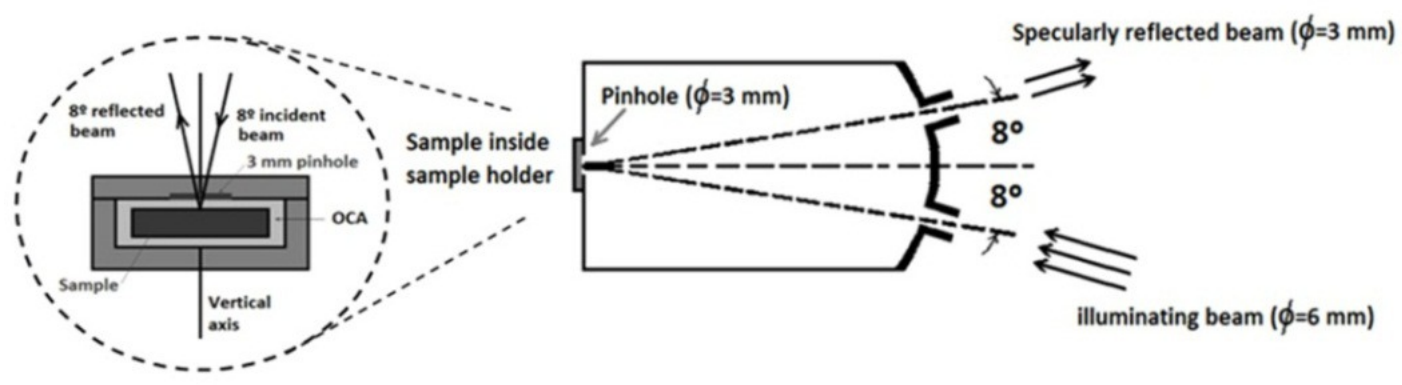

Figure 5: Specular reflectance measuring assembly.

For this setup, we consider the spectrum measured from the light source $\left(S_{s r}(\lambda)\right)$ and the specularly reflected spectra measured at any particular time $t$ from the sample $\left(R_{s r}(\lambda, \mathrm{t})\right)$. Using these spectra, we can calculate the $R_{s}$ spectrum of the sample at any time of the treatment with equation 4 :

$$
R_{s}(\lambda, t)=100 \% \times \frac{R_{s r}(\lambda, t)}{S_{s r}(\lambda)}
$$

With all the previously described measurements, we can calculate the absorbance and diffuse reflectance of the sample during the treatment. These calculations are presented in subsections 2.3.5 and 2.3.6.

\subsubsection{Absorbance calculation}

Considering the integrated measurements of $T_{t}$ and $R_{t}$ presented in equations 1 and 2 and assuming once again that the light spectrum that is used in the integrated measurements is $100 \%$, the absorbance of the sample can be determined as the difference between $100 \%$ of light used and the integrated measurements ${ }^{13}$.

Equation 5 shows the calculation of sample's absorbance spectrum at a time $t$ during the optical clearing treatment:

$$
A(\lambda, t)=100 \%-\left[T_{t}(\lambda, t)+R_{t}(\lambda, t)\right]
$$

\subsubsection{Diffuse reflectance calculation}

If we now consider that $R_{t}$ contains both specular and diffuse terms we can calculate $R_{d}$ from the measurements performed. This way, using the $R_{t}$ and $R_{s}$ measurements (performed in the same conditions), we can use equation 6 to calculate $R_{d}$ of the sample at any time $t$ of the treatment:

$$
R_{d}(\lambda, t)=R_{t}(\lambda, t)-R_{s}(\lambda, t)
$$

The results presented in all graphics of section 3 were calculated using all the six equations above.

\section{EXPERIMENTAL RESULTS}

Since the two studies were performed with EG, we will consider in this section each of the particular measurements and present the results obtained from the two types of samples side by side. This representation allows performing adequate comparison and immediate identification of the differences between the two cases.

We will start in the following subsection with the results of $T_{t}$, followed by the results of $T_{c}, R_{t}, R_{s}, A$ and $R_{d}$.

For each of the following subsections we will present three figures for each type of tissue sample (fresh or frozen). For the fresh samples, figures will always be presented on the left, while for frozen samples, figures will always be presented on the right. We will now make a brief explanation of each of these figures. 
For each of the two cases (fresh or frozen), the 3 figures will be accordingly to the following description:

In the first figure we present the corresponding spectrum for the "natural" sample (before adding the optical clearing agent).

$>$ The second figure shows the time-dependency lines for some particular wavelengths during the first minute of treatment. These lines have $1 \mathrm{sec}$ resolution in the time scale. The chosen wavelengths were $400 \mathrm{~nm}, 500 \mathrm{~nm}$, $600 \mathrm{~nm}, 700 \mathrm{~nm}, 800 \mathrm{~nm}, 900 \mathrm{~nm}$ and $1000 \mathrm{~nm}$.

$>$ Similarly to the second figure, the third figure shows the time-dependency curves for the same particular wavelengths $(400 \mathrm{~nm} \ldots . .1000 \mathrm{~nm}$ ), but now corresponding to 30 min of treatment and with one minute resolution.

This set of figures allows immediate identification of the "natural" characteristics of the muscle's response to light stimulations and comparison between both samples (fresh and frozen). On the other hand, the time dependence caused in the muscle's response to light can also be identified and compared easily.

By comparing between side-by-side figures, we can evaluate the differences between fresh and frozen samples. By comparing between the two cases, we can also identify the quality of the optical clearing effect created by EG and identify the advantages for each case. As we shall see by analyzing the figures below, both cases present advantages. Generally, the frozen samples show higher magnitude optical clearing effect, but its time dependence is not as well behaved as in the case of fresh samples. These facts are certainly related with the freezing process of the samples used in the second study for a period of $72 \mathrm{hrs}$ and consequently the optical clearing effect is not so straight-forward as in the case of fresh samples.

\subsection{Total transmittance}

The $T_{t}$ spectrum obtained from each sample before applying the immersion treatment in EG can be seen in both graphs of figure 6 :
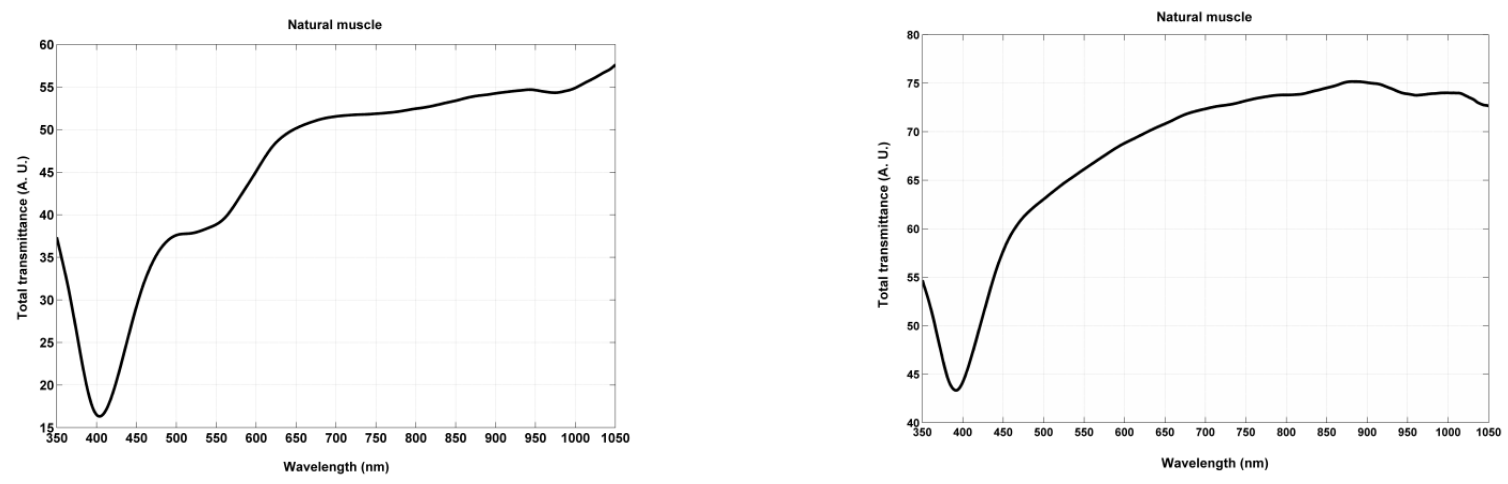

Figure 6: Total transmittance spectrum from natural fresh muscle (left) and from natural frozen muscle (right).

We can see from figure 6 that in general the $T_{t}$ spectrum is similar between both cases, but some differences can be identified. By comparing both graphs, we can see different levels of $T_{t}$ between fresh and frozen sample. Additionally, for the frozen sample we cannot see the myoglobin Q-bands $(544$ to $582 \mathrm{~nm}){ }^{14}$, possibly due to a loss of myoglobin during the freezing time.

During the treatment time with EG, we have performed sequential measurements of $T_{t}$. In figure 7 we represent the evolution of $T_{t}$ during the first minute of treatment for both samples: 

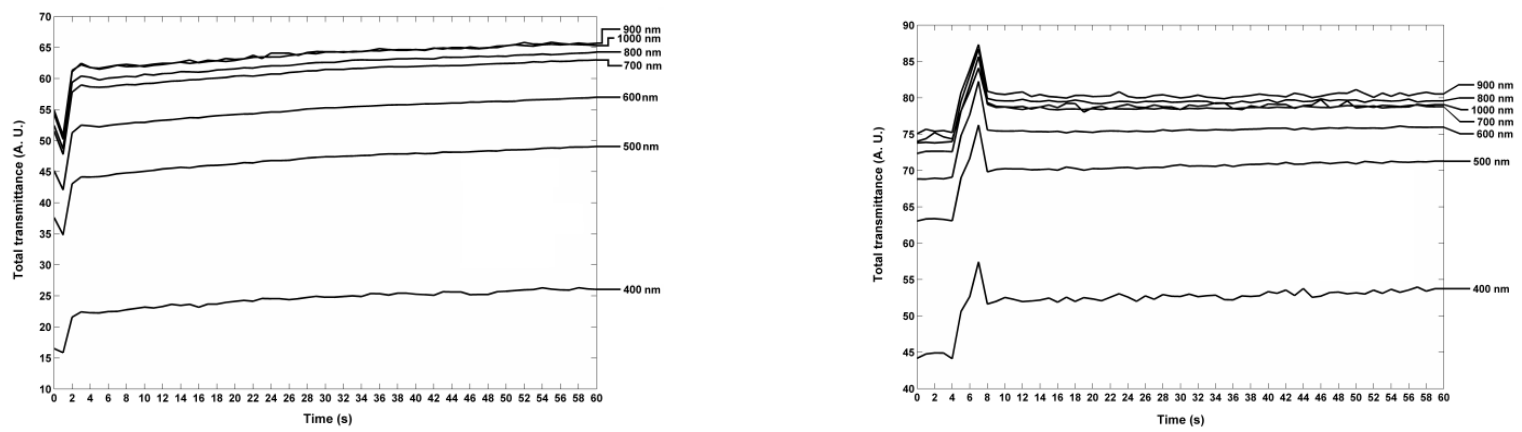

Figure 7: Total transmittance evolution in the first minute of treatment for some wavelengths. Treatment of fresh sample (left) and frozen sample (right).

Comparing between graphs in figure 7, we see that the frozen sample presents a peak in $T_{t}$ at $7 \mathrm{sec}$ and it shows a smaller rise than the fresh sample during the first minute of treatment with EG.

Figure 8 represents the same variation with time, but now for 30 min of treatment and with 1 min resolution:
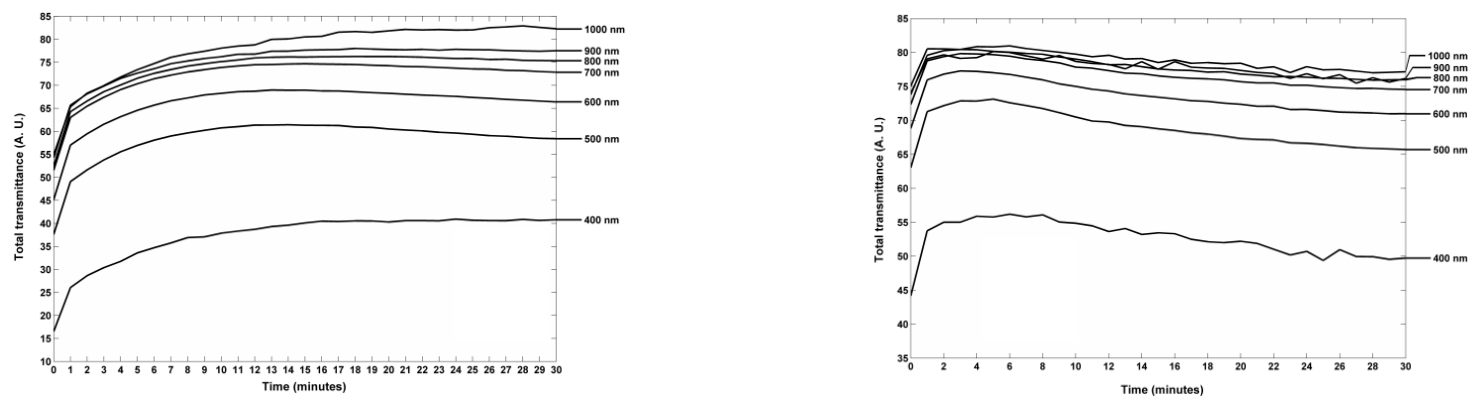

Figure 8: Total transmittance evolution during the treatment for some particular wavelengths. Treatment of fresh sample (left) and frozen sample (right).

Comparing between graphs in figure 8, we see that the fresh sample presents an increasing response during almost all treatment. Only during the saturation regime, which begins at 13 min of treatment, we observe a little decreasing behavior for some individual wavelengths. In the case of the frozen sample, the saturation regime begins at $10 \mathrm{~min}$ and it shows a decreasing behavior for all wavelengths, indicating that the sample expels part of the agent that was strongly inserted at the beginning to achieve equilibrium.

\subsection{Collimated transmittance}

Using the $T_{c}$ assembly presented in figure 4 , we have made the necessary measurements to obtain the $T_{c}$ spectra during the treatments. Figure 9 shows the $T_{c}$ spectrum of both samples before applying the immersion treatment with EG.
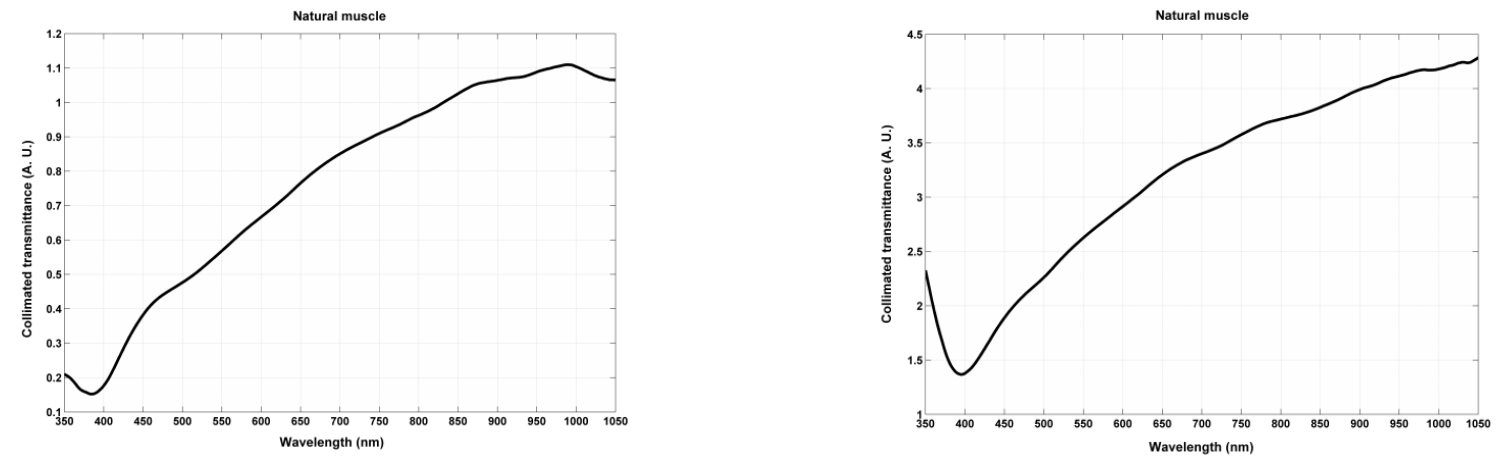

Figure 9: Collimated transmittance spectrum from natural fresh muscle (left) and from natural frozen muscle (right). 
By comparing between graphs of figure 9 we observe similarity in the form of the collimated spectra. Both spectra are typical for fibrous tissue, $T_{c}$ rises with wavelength. The case of frozen sample presents higher $T_{c}$. The myoglobin Soret band (around $400 \mathrm{~nm}$ ) is better defined for the frozen sample and the Q-bands are almost not visible in the fresh sample and not visible at all for the frozen sample. Such fact is probably related to a loss of myoglobin during the freezing process, as already explained in the case to figure 6.

Figure 10 shows the evolution of $T_{c}$ during the first minute of treatment for the usual wavelengths:
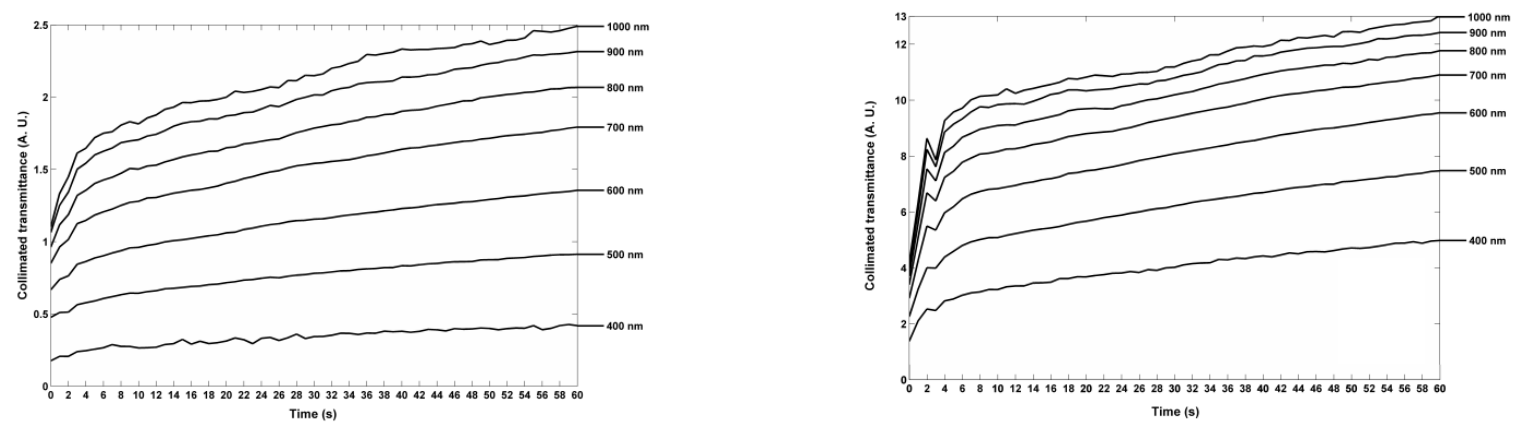

Figure 10: Collimated transmittance evolution in the first minute of treatment for some particular wavelengths. Treatment of fresh sample (left) and frozen sample (right).

From the graphs in figure 10, we see the similarity in the behavior of the optical clearing effect during the first minute. The frozen sample has higher magnitude of natural $T_{c}$ and it presents a bigger increase than the fresh sample during the treatment. A small oscillation is seen in the case of the frozen sample around $3 \mathrm{~min}$, which is more visible for higher wavelengths. Such oscillation might be related to a dehydration stage of the sample.

In figure 11 we present the evolution of $T_{c}$ observed for both samples during 30 minutes of treatment:
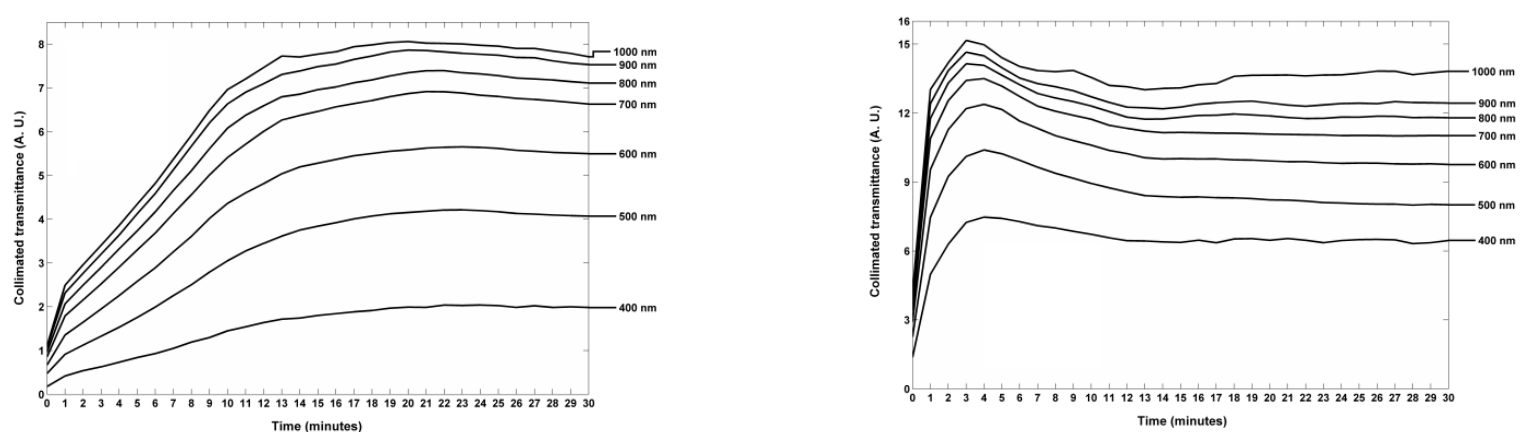

Figure 11: Collimated transmittance evolution during the treatment for some particular wavelengths. Treatment of fresh sample (left) and frozen sample (right).

We can see from figure 11 that the long-time variation of $T_{c}$ is different between both cases. In the case of the fresh sample (left graph of figure 11), we see that the initial EG-induced strong diffusion of water is happened in two stages which correspond to: 1-strong initial rise during the first minute, and 2-less intense strong rising behavior between 1 and $10 \mathrm{~min}$. After $10 \mathrm{~min}$ of treatment $T_{c}$ tends to slow its rising behavior between 10 and $20 \mathrm{~min}$. The saturation regime begins at approximately $20 \mathrm{~min}$ and it shows a slight decreasing behavior, indicating that within the tissue sample some equilibrium state between water flux and EG flux was established. The slight tissue "darkening" can be a consequence of some impact of the agent on the tissue. For instance, some tissue swelling may occur ${ }^{9}$.

In the case of the frozen sample (right graph of figure 11), we see an initial strong rise of $T_{c}$, restrained to the first minute. After that $T_{c}$ rises a little further in the next $3 \mathrm{~min}$ and then it begins to decrease to enter an almost constant level saturation regime. By comparing between both cases, we see that $T_{c}$ rises differently in the two samples. After $30 \mathrm{~min}$ of 
treatment, we can say that for the fresh sample $T_{c}$ has an 8 -fold increase and for the frozen sample it shows only 5 -fold increase (approximated values).

We see from this analysis that the freezing process influences EG interaction with the muscle in terms of behavior and magnitude of the optical clearing effect created.

\subsection{Total reflectance}

Using the measuring setup assembly represented in figure 3, we have measured spectra to calculate the $R_{t}$ of the muscle in natural state and during treatment. Figure 12 shows the $R_{t}$ spectra of both natural samples (fresh and frozen, respectively).
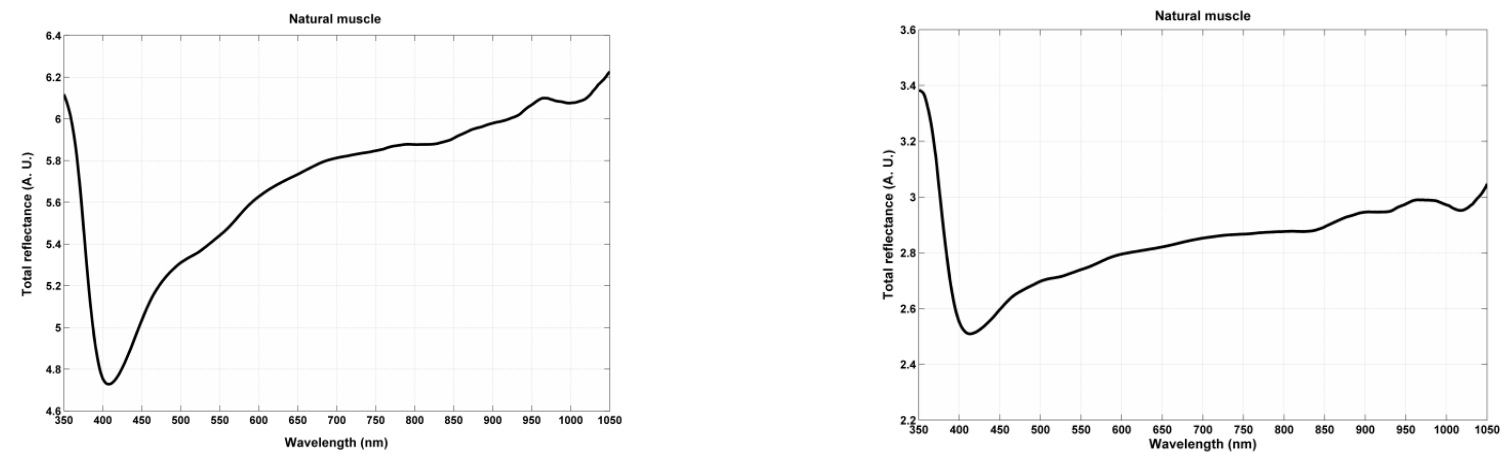

Figure 12: Total reflectance spectrum from natural fresh muscle (left) and from natural frozen muscle (right).

In this case, we see that the fresh sample presents a higher level of $R_{t}$ than for the frozen sample. We see also that the magnitude of the Soret band is different between cases.

In figure 13 we present the time variation of $R_{t}$ for both samples during the first minute of treatment with EG for the selected wavelengths:
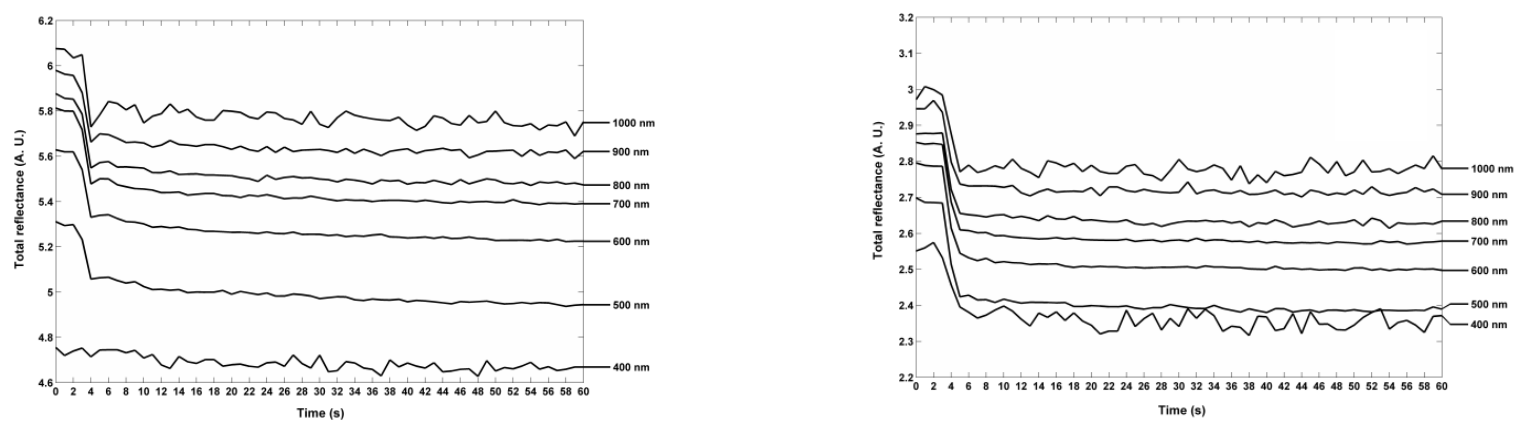

Figure 13: Total reflectance evolution in the first minute of treatment for some particular wavelengths. Treatment of fresh sample (left) and frozen sample (right).

From comparison between graphs in figure 13, we see great similarity in the time dependence of $R_{t}$ between samples. On the other hand, the levels verified for the different wavelengths are different between cases, indicating that the frozen sample has an initial smaller total reflectance. We see that the major decrease in $R_{t}$ occurs within the first $5 \mathrm{sec}$ in both cases.

Now considering the long time variation of $R_{t}$, we have represented in figure 14 the evolution for the selected wavelengths: 

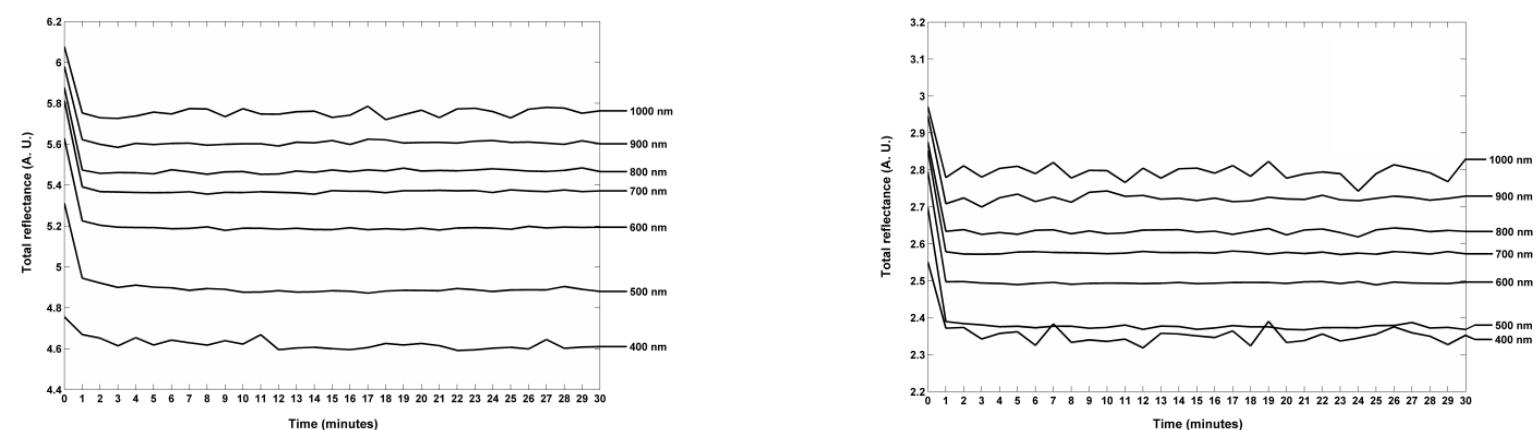

Figure 14: Total reflectance evolution during the treatment for some particular wavelengths. Treatment of fresh sample (left) and frozen sample (right).

By comparing between graphs of figure 14 and apart from the different levels of $R_{t}$ observed, we see that the time dependence during the treatment is similar for both samples: a strong decrease of $R_{t}$ in the first minute, tending after that to remain constant during the rest of the treatment.

\subsection{Specular reflectance}

Using the experimental assembly represented in figure 5 we have measured the spectra to calculate $R_{S}$ of the samples during the treatments with equation 4 . Figure 15 shows the $R_{S}$ spectra of both samples before treatment was applied.
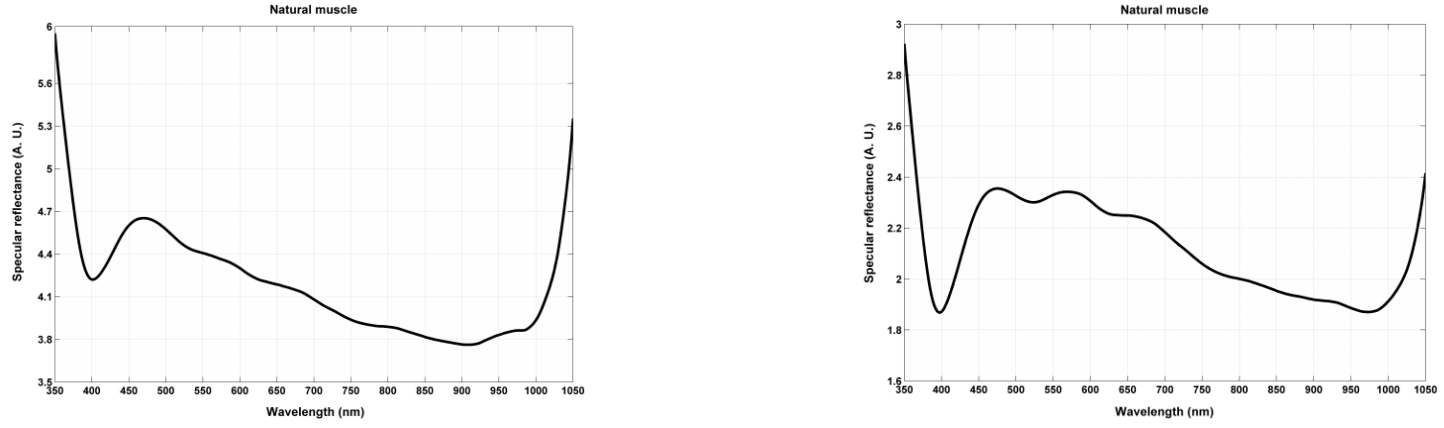

Figure 15: Specular reflectance spectrum from natural fresh muscle (left) and natural frozen muscle (right).

Comparing between the spectra represented in figure 15, we see that the frozen sample shows smaller levels of $R_{s}$ for all wavelengths. The form of the $R_{S}$ spectrum is very similar between the two cases $-R_{s}$ decreases with wavelength in the visible range. The only differences are that the frozen sample presents some small amplitude peaks between 500 and 700 $\mathrm{nm}$ and the magnitudes of the Soret band are different between samples.

Considering now the optical clearing treatment, and in particular the first minute of treatment of both samples, we can see in figure 16 the evolution of $R_{s}$ for the selected wavelengths:
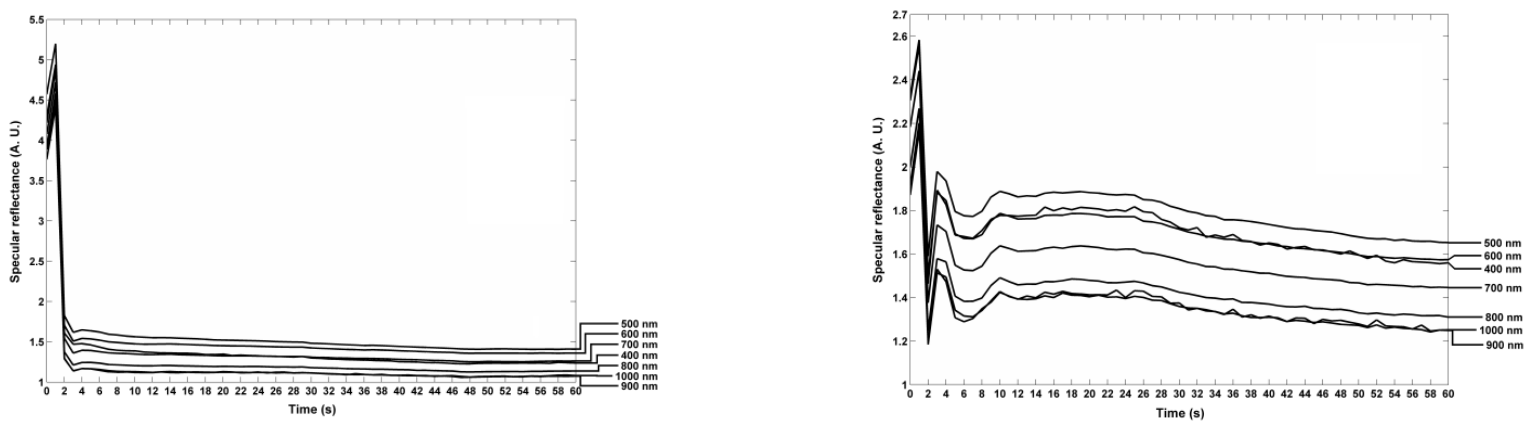

Figure 16: Specular reflectance evolution in the first minute of treatment for some particular wavelengths. Treatment of fresh sample (left) and frozen sample (right). 
From graphs in figure 16 we see that the frozen sample produces some oscillations in the first seconds of treatment. We can also see that the initial rise of $R_{S}$ is stronger for the fresh sample, but the levels in the saturation regime are similar between cases. The initial rise in the treatment of the fresh sample is certainly related to transit processes within the interfaces between tissue surface and optical clearing agent in the course of filling up the sample holder with the agent. Such initial increase of specular reflectance (seen for both samples) might also be related to a less tissue surface roughness that is caused by filling up of surface inhomogeneities by EG and immediate loss of some water by superficial layers of tissue, caused by EG osmotic pressure. We verify also for both cases that after the initial rise, $R_{s}$ decreases strongly between 1 and 2 seconds of treatment. This behavior indicates a strong water flux from superficial layers of the sample induced by EG during the first few seconds. After that, a slow decreasing behavior can be observed after 6 sec for the fresh sample and after $26 \mathrm{sec}$ for the frozen sample, indicating that the lower tissue layers have a much lesser influence on the measured specular reflectance. The interval between 2 and 26 seconds for the frozen sample, where we can see some oscillatory behavior (right graph of figure 16), indicates the occurrence of different stages of tissue dehydration.

Considering now the total time of treatment, we present in figure 17 the evolution of $R_{s}$ for both samples:
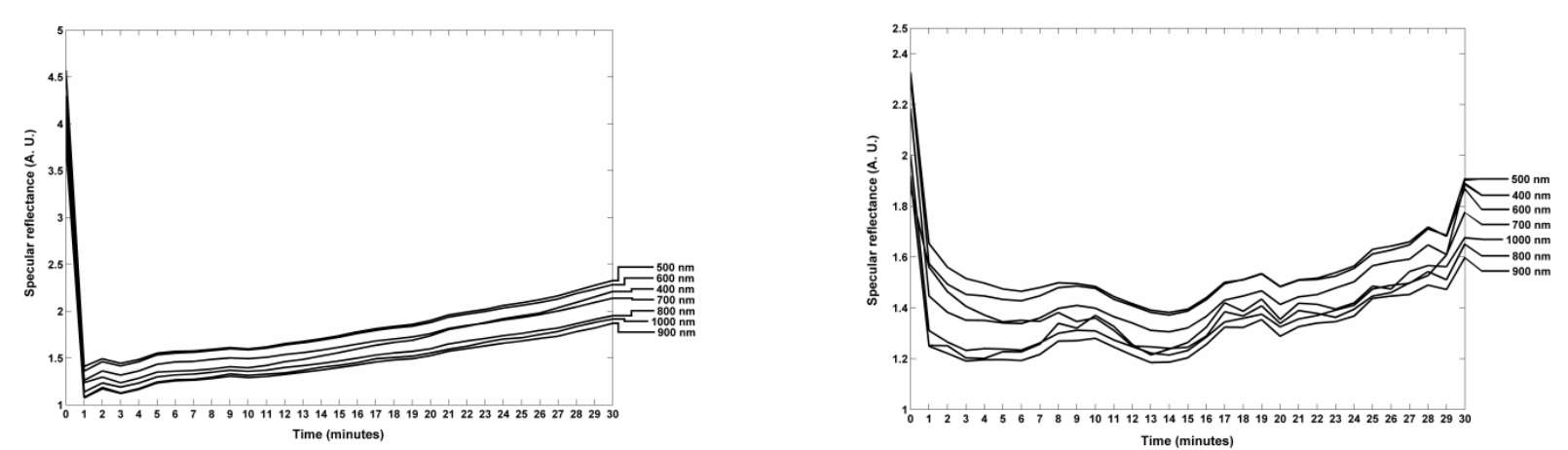

Figure 17: Specular reflectance evolution during the treatment for some particular wavelengths. Treatment of fresh sample (left) and frozen sample (right).

The graphs in figure 17 show similarity in behavior - a strong decrease during the first minute and a little increasing saturation regime after that. The behavior observed for the frozen sample is not as good as the one observed for the fresh sample; it presents some oscillations during the saturation regime. For the treatment of the fresh sample, we see a major decrease in $R_{s}$ within the first minute. After that, the saturation regime is observed with an increasing behavior, which indicates that the upper layers of the sample are losing some of the agent. Nevertheless, even after 30 min of treatment, we can still see levels of $R_{s}$ that are significantly lower than in the natural muscle. The oscillatory behavior seen in the saturation regime for the frozen sample (right graph of figure 17) indicates that the sample suffers dehydration (and possibly EG insertion) in different periods of the treatment. Such different stages of tissue dehydration might be related to tissue structure modification during the freezing process. The inner layers of the tissue might be still defreezing during the treatment, causing different stages of dehydration.

\subsection{Absorbance}

Considering the measurements of $T_{t}$ and $R_{t}$ presented in previous subsections, we have used equation 5 to calculate the sequential absorbance spectra for the treatments of the fresh and frozen samples with EG. In figure 18 we present the calculated absorbance spectrum for the "natural" state of both samples: 

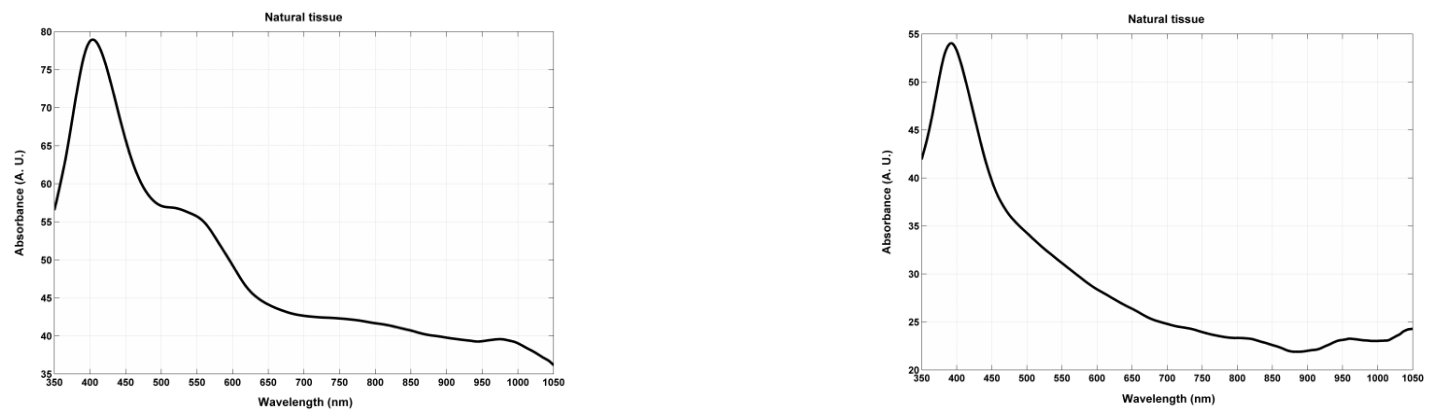

Figure 18: Absorbance spectrum from natural fresh muscle (left) and natural frozen muscle (right).

By comparing between spectra in figure 18, we see that the absorbance levels are different: the fresh sample shows higher absorbance than the frozen sample. The spectral form is similar, but the frozen sample does not show the myoglobin Q-bands.

During the first minute of treatment with EG we have observed the following variations in absorbance for the usual wavelengths:
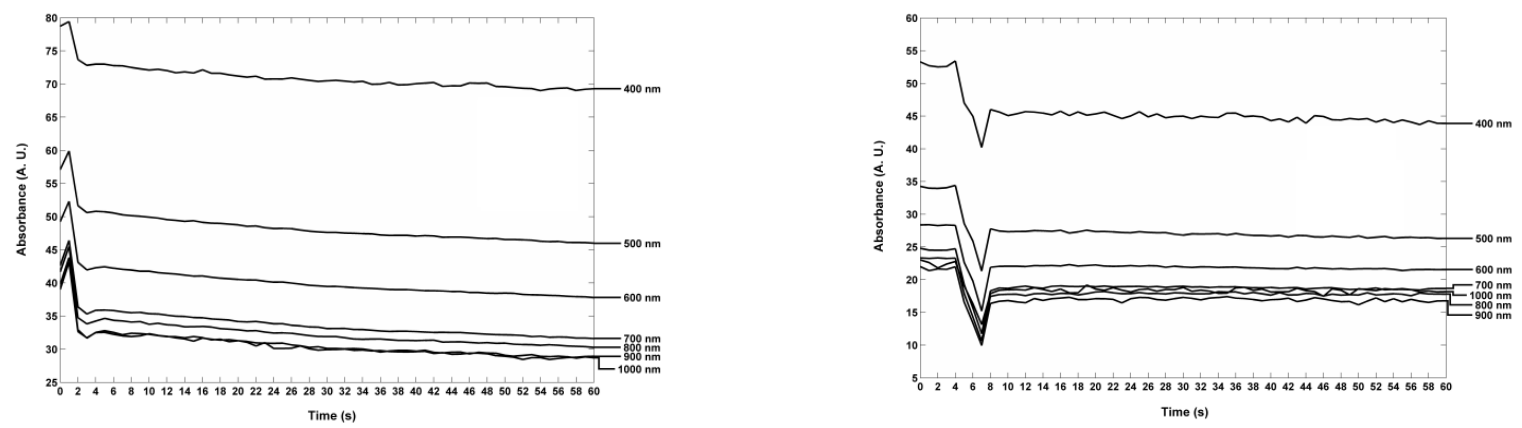

Figure 19: Absorbance evolution in the first minute of treatment for some particular wavelengths. Treatment of fresh sample (left) and frozen sample (right).

From graphs in figure 19, we see that only the frozen sample shows an oscillation around 7 sec. Additionally, the absorbance levels are smaller for the frozen sample and the decreasing behavior is more intense in the case of the fresh sample. During this first minute of treatment, the overall behavior indicates a decrease in absorbance for both samples, but the decrease in the case of the fresh sample is stronger than the one seen for the frozen sample.

For the 30 min of treatment, we present in figure 20 the evolution of absorbance for both samples:
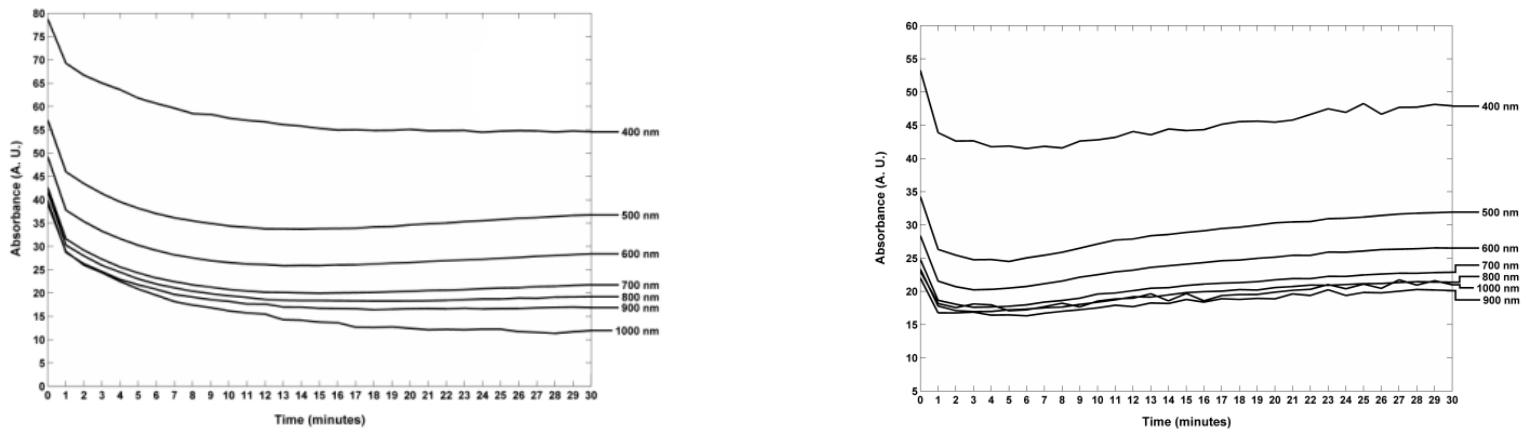

Figure 20: Absorbance evolution during the treatment for some particular wavelengths. Treatment of fresh sample (left) and frozen sample (right). 
Comparing between graphs in figure 20, we observe that the absorbance shows different saturation regimes when treated with EG: in the case of the fresh sample it is almost constant, but in the case of the frozen sample it is significantly increasing with time. The overall decrease seen in the case of the fresh sample is higher in magnitude than the one seen for the frozen sample. Nevertheless, we can see that in both samples absorbance decrease from the natural stage during the treatment. Such decrease indicates also a decrease in the light absorption by both muscle samples, because of a less scattering in the system. It is known that for a scattering tissue the absorbed light energy strongly depends on the scattering coefficient. When the tissue becomes clear, photons have shorter paths in the tissue and consequently have less probability of being absorbed.

\subsection{Diffuse reflectance}

To finalize this comparative study, we present the calculated $R_{d}$. Figure 21 shows the natural $R_{d}$ spectrum for both samples.
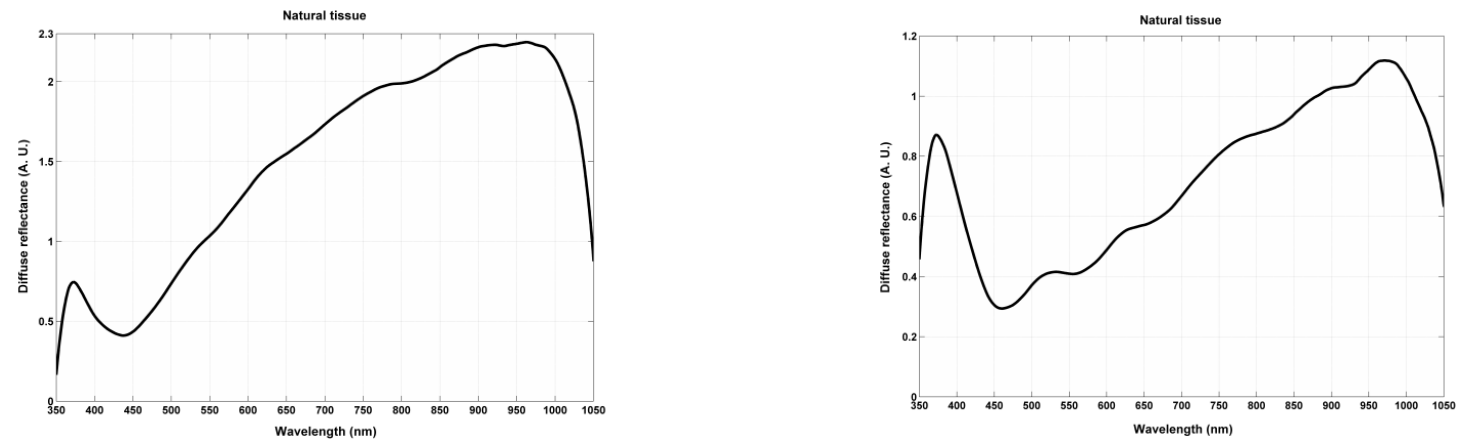

Figure 21: Diffuse reflectance spectrum from natural fresh muscle (left) and natural frozen muscle (right).

We can see a similar spectral form between the two cases, but there are some particular differences: The $R_{d}$ levels are different, different magnitude peaks at lower wavelengths and some spectral oscillations at middle wavelengths for the frozen sample. These oscillations are caused by the oscillations seen in the measurements of $R_{s}$ (see figure 15).

Figure 22 presents the evolution of $R_{d}$ for both samples during the first minute of treatment:
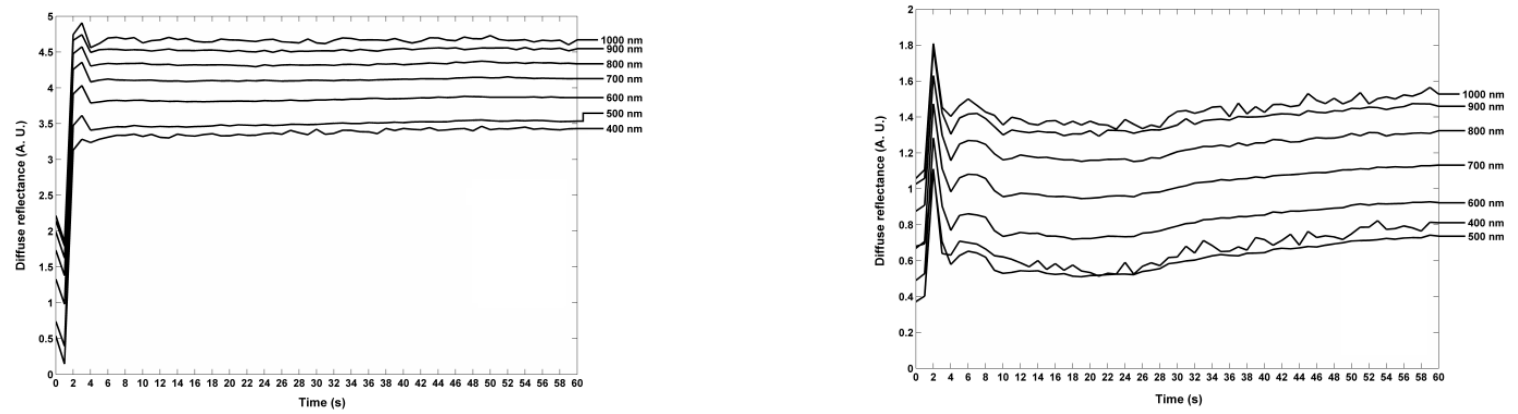

Figure 22: Diffuse reflectance evolution in the first minute of treatment for some particular wavelengths. Treatment of fresh sample (left) and frozen sample (right).

By comparing between graphs in figure 22 we see that the fresh sample shows an almost constant behavior after the fourth minute (well above natural values), which is not verified for the frozen sample. The frozen sample shows a little increasing behavior after the initial oscillation. From analysis of both graphs in figure 22, we see a major increase in $R_{d}$ before $5 \mathrm{sec}$ for the fresh sample and before $3 \mathrm{sec}$ for the frozen sample. For the fresh sample, and after this initial increase, we observe a stabilization of the $R_{d}$ levels for the remaining of the first minute of treatment. This fact indicates that the possible changes in the internal structure of the muscle are not significant to change drastically the diffuse reflectance of the sample. For the case of the frozen sample (right graph of figure 22), we see a little decrease between 3 and $4 \mathrm{sec}$ of treatment, followed by an oscillation before entering the slow linearly increase for the remaining of the first 
minute. Such behavior is a result of using the measurements of $R_{s}$, which show a similarly oscillatory behavior (see figure 16 - right graph).

Figure 23 shows the evolution of $R_{d}$ during the 30 min of treatment:
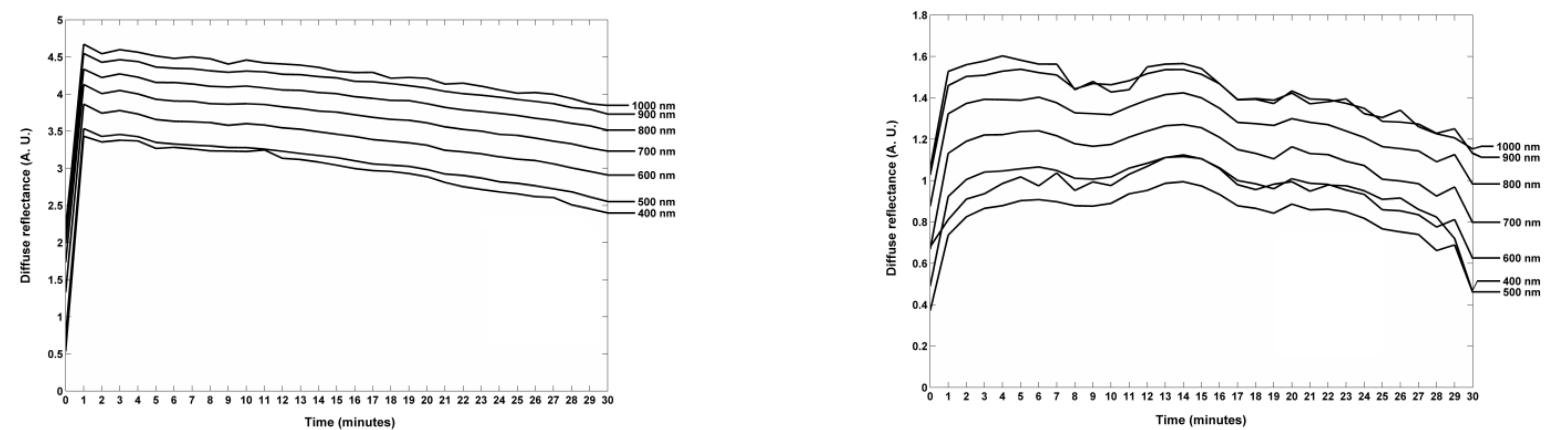

Figure 23: Diffuse reflectance evolution during the treatment for some particular wavelengths. Treatment of fresh sample (left) and frozen sample (right).

When comparing between graphs in figure 23 we see that both cases show a decreasing behavior during saturation regime, but the frozen sample presents oscillations, which is not the case for the fresh sample. Once more, and due to the fact of using $R_{t}$ and $R_{s}$ to calculate $R_{d}$, we can justify the occurrence of the oscillations for the frozen sample by the occurrence of oscillations in the measurements of $R_{s}$ (see figure 17 - right graph). Following the description already presented for specular reflectance regarding refractive index mismatch at interface between EG and tissue and initial transient effects at optical clearing, we can only consider the beginning of diffuse reflectance variation after 1 min of treatment for both cases. This way, when interpreting the graphs in figure 23, we should neglect the first minute of treatment in both cases. Assuming this, we verify that for both samples, $R_{d}$ decreases with time in a more or less linearly manner. Such decrease is due to the high diffusion power of EG. The frozen sample presents some oscillations during the saturation regime, which was already explained in the analysis of the $R_{s}$ measurements.

\subsection{Calculation of the diffusion coefficient of EG}

As a complement to the studies performed above, we will now estimate diffusion time and diffusion coefficient associated with EG diffusion into and induced by EG action water diffusion out from tissue sample.

Considering the fresh sample and the measurements of collimated transmittance presented above (figure 11 - left graph), we can adjust the $T_{c}$ measurements to an exponential curve like the one presented in equation 7 to determine the diffusion time as ${ }^{9}$ :

$$
T_{c}(\lambda, t)=a \times\left[1-\exp \left(-\frac{t}{\tau(\lambda)}\right)\right]
$$

Figure 24 shows the $T_{c}$ data for the usual wavelengths and corresponding adjustments according to equation 7 : 


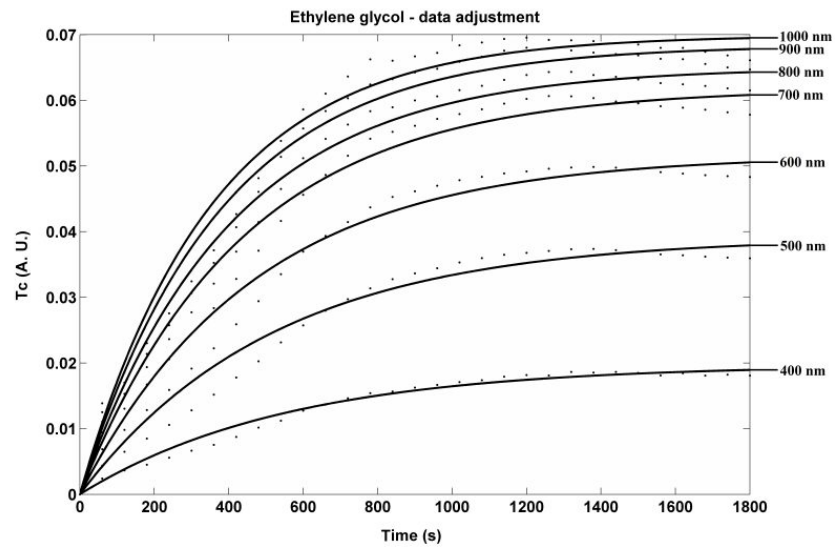

Figure 24: Data fitting using equation 7 for $T_{c}$ experimental measurements from fresh sample.

The parameters determined from the individual adjustments made in figure 24 , are represented in table 1:

Table 1: Fitting parameters for curves in figure 24.

\begin{tabular}{|c||c|c|c||c|c|c|}
\hline \multirow{2}{*}{$\begin{array}{c}\text { Wavelength } \\
(\mathrm{nm})\end{array}$} & $\begin{array}{c}\text { a (mean) } \\
\text { (A.U.) }\end{array}$ & $\begin{array}{c}\text { Minimum } \\
\text { (A.U.) }\end{array}$ & $\begin{array}{c}\text { Maximum } \\
(\text { A.U.) }\end{array}$ & $\begin{array}{c}\tau(\text { mean }) \\
(\mathrm{s})\end{array}$ & $\begin{array}{c}\text { Minimum } \\
(\mathrm{s})\end{array}$ & $\begin{array}{c}\text { Maximum } \\
(\mathrm{s})\end{array}$ \\
\hline$\lambda=400$ & 0.01972 & 0.01905 & 0.02039 & 556.2 & 491.1 & 621.3 \\
\hline$\lambda=500$ & 0.03916 & 0.03764 & 0.04068 & 522.4 & 447.8 & 597 \\
\hline$\lambda=600$ & 0.05165 & 0.05011 & 0.05319 & 467.6 & 409.8 & 525.4 \\
\hline$\lambda=700$ & 0.06181 & 0.06008 & 0.06355 & 435.9 & 381.3 & 490.4 \\
\hline$\lambda=800$ & 0.06503 & 0.06353 & 0.06654 & 402.2 & 356.8 & 447.5 \\
\hline$\lambda=900$ & 0.06838 & 0.06688 & 0.06988 & 375.8 & 332.5 & 419 \\
\hline$\lambda=1000$ & 0.06992 & 0.0684 & 0.07144 & 355 & 311.9 & 398.1 \\
\hline
\end{tabular}

From the data presented in table 1 , we obtain the average diffusion time for the fresh sample: $\tau=445.0 \mathrm{~s}$. Such value has an associated standard deviation of 74.9. With this value and with the thickness value that we have measured for this time of treatment from a muscle sample under treatment with EG, we can use equation 8 to calculate the diffusion coefficient as ${ }^{9}$ :

$$
\tau=\frac{d^{2}}{\pi^{2} \times D_{a}}
$$

Using the diffusion mean time under EG action in the muscle and the measured thickness of the sample for that time of treatment $(0.0288 \mathrm{~cm})$, we have calculated the diffusion coefficient in the muscle. Such calculation is presented in equation 9:

$$
D_{E G}=\frac{d_{E G}^{2}}{\pi^{2} \times \tau_{E G}}=\frac{0.0288^{2}}{\pi^{2} \times 445.0}=1.89 \times 10^{-7} \mathrm{~cm}^{2} / \mathrm{s}
$$

Now, for the case of the frozen sample and considering the $T_{c}$ measurements represented in figure 11 (right side), we have performed the same methodology to estimate the diffusion time and consecutively the diffusion coefficient. Considering equation 7 above, we have made adjustments to the $T_{c}$ data for each wavelength. Such data and adjustment curves are represented in figure 25 : 


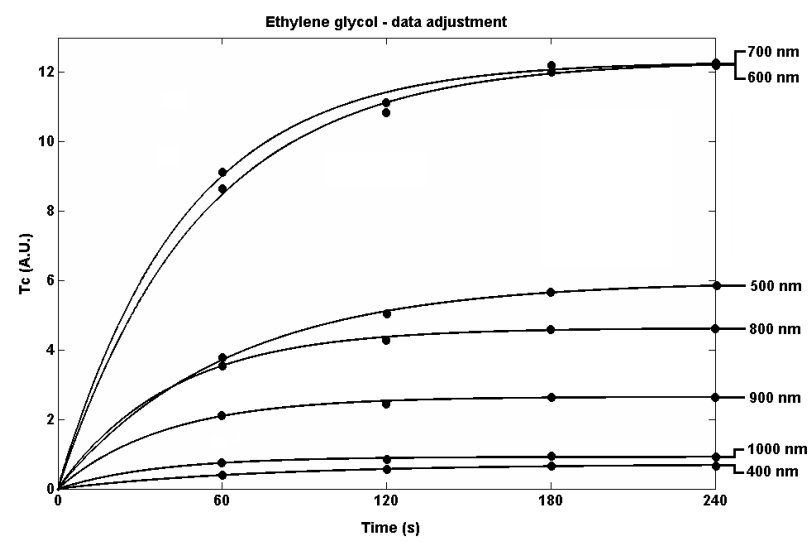

Figure 25: Data fitting using equation 7 for $T_{c}$ experimental measurements from frozen sample.

The parameters determined from the individual adjustments made in figure 25 , are represented in table 2:

Table 2: Fitting parameters for curves in figure 25.

\begin{tabular}{|c||c|c|c||c|c|c|}
\hline \multirow{2}{*}{$\begin{array}{c}\text { Wavelength } \\
(\mathrm{nm})\end{array}$} & $\begin{array}{c}\text { a (mean) } \\
\text { (A.U.) }\end{array}$ & $\begin{array}{c}\text { Minimum } \\
\text { (A.U.) }\end{array}$ & $\begin{array}{c}\text { Maximum } \\
\text { (A.U.) }\end{array}$ & $\begin{array}{c}\tau(\text { mean }) \\
(\mathrm{s})\end{array}$ & $\begin{array}{c}\text { Minimum } \\
(\mathrm{s})\end{array}$ & $\begin{array}{c}\text { Maximum } \\
(\mathrm{s})\end{array}$ \\
\hline$\lambda=400$ & 0.7101 & 0.6579 & 0.7622 & 74.46 & 57.95 & 90.98 \\
\hline$\lambda=500$ & 5.977 & 5.804 & 6.15 & 61.67 & 55.43 & 67.92 \\
\hline$\lambda=600$ & 12.34 & 11.85 & 12.84 & 51.62 & 43.22 & 60.01 \\
\hline$\lambda=700$ & 12.31 & 11.81 & 12.80 & 45.52 & 37.27 & 53.76 \\
\hline$\lambda=800$ & 4.63 & 4.448 & 4.811 & 41.25 & 33.34 & 49.16 \\
\hline$\lambda=900$ & 2.649 & 2.544 & 2.753 & 38.24 & 30.3 & 46.17 \\
\hline$\lambda=1000$ & 0.9243 & 0.8714 & 0.9772 & 36.25 & 24.75 & 47.76 \\
\hline
\end{tabular}

Considering the adjustment data presented in table 2, we calculated the average diffusion time for the frozen sample as $49.86 \mathrm{~s}$. The standard deviation for the calculated tau values is 13.91. Considering the thickness measurement made from the fresh sample at a time of treatment corresponding to the mean tau for the frozen sample as the best possible estimation available for the present case, we can use equation 8 to calculate the diffusion coefficient. Such calculation with equation 8 is represented in equation 10:

$$
D_{E G}(\text { frozen })=\frac{0.0426^{2}}{\pi^{2} \times 49.86}=3.69 \times 10^{-6} \mathrm{~cm}^{2} / \mathrm{s}
$$

Such calculation is not precise due to the use of a thickness value measured from the fresh sample, instead from the frozen sample, but it represents the best possible estimation. By comparing both the diffusion coefficients calculated for the fresh and the frozen samples, we observe that the case of the frozen sample presents a diffusion for the combined fluxes of EG into and water out faster that the case of the fresh sample. Such difference is mainly imposed by the fast dehydration that the frozen sample suffers due to the osmotic pressure when the treatment is initiated.

\section{CONCLUSIONS}

We have used fresh and frozen samples from the abdominal wall muscle of rat to study and compare the optical responses of those samples to light stimulation during treatment with ethylene glycol.

We have verified that the frozen sample presents higher levels of total and collimated transmittance and also smaller levels of reflectance (total, specular and diffuse) and absorbance than the fresh sample. On the other hand, if we consider the natural spectra for the cases of total transmittance, collimated transmittance, total reflectance and absorbance, we see 
that the fact of freezing the sample for 72 hours removes some spectral signatures (myoglobin Q-bands). In the case of the natural specular and diffuse reflectance spectra, we see the appearance of some new spectral signatures in the case of the frozen sample that were not visible for the fresh sample.

Considering the optical clearing effects created by ethylene glycol presented for both cases, some significant differences could be identified. In opposition to the case of the fresh sample, we have registered the appearance of oscillations in the first seconds of treatment for the frozen sample in the cases of total transmittance, collimated transmittance, specular reflectance, absorbance and diffuse reflectance. In the case of total reflectance, oscillations appear in both cases studied (fresh and frozen). If we consider the total time of treatment, we observe great similarity between the two cases.

One of the differences observed is that due to higher levels of transmittance (total and collimated) and lower levels of reflectance (total, specular and diffuse) for the frozen sample, the optical clearing effect created by immersing the tissue in ethylene glycol shows higher levels of transmittance and lower levels of reflectance along the time for the frozen sample. In the case of absorbance, we have observed that the fresh sample presents a decreasing absorbance during all treatment time, while the frozen sample presents an initial decrease and an increasing saturation regime after approximately 6 or $7 \mathrm{~min}$.

By analyzing the comparisons made in the different measurements between fresh and frozen samples, we can recognize that the freezing process is responsible for significant changes in the interaction of the muscle with light. One of the changes is that for some of the measurements presented we see differences between the "natural" spectra of fresh and frozen samples. On the other hand, in some of the cases presented, we could see the appearance of oscillations in the case of the frozen sample during the optical clearing treatment with ethylene glycol.

\section{ACKNOWLEDGEMENTS}

The authors would like to thank the following institutions for all the help in preparation of the samples and resources made available to perform the measurements:

- CIETI - Centro de Inovação em Engenharia e Tecnologia Industrial, ISEP - Instituto Superior de Engenharia do Porto, Portugal.

- LAIMM - Laboratório de Apoio à Investigação em Medicina Molecular, Departamento de Biologia Experimental, Faculdade de Medicina da Universidade do Porto, Portugal.

This work was also supported in part by grants: RFBR 11-02-00560-a, 12-02-92610-KO_a; 224014 PHOTONICS4LIFE of FP7-ICT-2007-2; 1.4.09 of RF Ministry of Education and Science; RF Governmental contracts 11.519.11.2035, 14.B37.21.0728, and 14.B37.21.0563; FiDiPro, TEKES Program (40111/11), Finland; SCOPES EC, Uzb/Switz/RF, Swiss NSF, IZ74ZO 137423/1; RF President’s grant “Scientific Schools”, 1177.2012.2.

\section{REFERENCES}

[1] Bashkatov, A. N., Genina, E. A., Tuchin, V. V., "Optical properties of skin, subcutaneous, and muscle tissues: a review," Journal of Innovative optical Health Sciences 4(1), 9-38 (2011).

[2] Plotnikov, S., Juneja, V., Isaacson, A. B., Mohler, W., Campagnola, P., "Optical clearing for improved contrast in second harmonic generation imaging of skeletal muscle," Biophysical Journal 90(1), 328-339 (2006).

[3] laComb, R., Nadiarnykh, O., Carey, S., Campagnola, P., "Quantitative second harmonic generation imaging and modeling of the optical clearing mechanism in striated muscle and tendon," Journal of Biomedical Optics 13(2), 2110921111 (2008).

[4] Rylander, C. G., Stumpp, O. F., Milner, T. E., Kemp, N. J., Mendenhall, J. M., Diller, K. R., Welch, A. J., "Dehydration mechanism of optical clearing in tissue," Journal of Biomedical Optics 11(4), 041117-041117-7 (2006).

[5] Oliveira, L., [Study of the spectral transmission response of biological tissues under the influence of different osmotic agents], MsC thesis, FEUP - Faculdade de Engenharia da Universidade do Porto, Porto, Portugal (2007). 
[6] Reinoso, R. F., Telfer, B. A., Rowland, M., “Tissue water content in rats measured by desiccation,” J. Pharmacol. Toxicol. Methods 38(2), 87-92 (1997).

[7] Oliveira, L., Lage, A., Pais Clemente, M., Tuchin, V.V., "Rat muscle opacity decrease due to the osmosis of a simple mixture," Journal of Biomedical Optics 15(5), 055004 - 055004-9 (2010).

[8] Oliveira, L. M., Lage, A. S., Pais Clemente M. and Tuchin, V. V., "Concentration dependence of the optical clearing effect created in muscle immersed in glycerol and ethylene glycol," Proc. SPIE 6535, 653511 (2007).

[9] Tuchin, V. V., [Optical Clearing of Tissues and Blood], SPIE Press, Bellingham, WA (2006).

[10] Hirshburg, J., Choi, B., Nelson, J. S., Yeh, A. T., "Collagen solubility correlates with skin optical clearing," Journal of Biomedical Optics 11(4), 040501 (2006).

[11] Genina, E. A., Bashkatov, A. N., Tuchin, V. V., “Tissue optical immersion clearing,” Expert Rev. Med. Devices 7(6), 825-842, (2010).

[12] Cicchi, R., Pavone, F. S., Massi, D., Sampson, D. D., "Contrast and Depth enhancement in two-photon microscopy of human skin ex vivo by use of optical clearing agents," Optics Express 13(7), (2005).

[13] Bass, M., Mahajan, V. N., Van Stryland, E., [Handbook of Optics - Vol. II], McGraw Hill, (2010).

[14] Bowen, W. J., "The absorption spectra and extinction coefficients of myoglobin," J. Biol. Chem., 179, 235-245 (1949). 